\title{
Semi-Oblivious Traffic Engineering with SMORE
}

\author{
Praveen Kumar \\ Cornell University \\ praveenk@cs.cornell.edu \\ Nate Foster \\ Cornell University \\ jnfoster@cs.cornell.edu
}

\author{
Yang Yuan \\ Cornell University \\ yy528@cornell.edu \\ Robert Kleinberg
Cornell University
rdk@cs.cornell.edu
}

\author{
Chris Yu \\ CMU \\ christoy@cs.cmu.edu \\ Petr Lapukhov \\ Facebook \\ petr@fb.com
}

\section{Chiun Lin Lim \\ Facebook \\ chiunlin@fb.com}

\begin{abstract}
Wide-area networks are expected to meet the competing objectives of high performance and reliability in the presence of various operational constraints and failures. Operators of such networks use traffic engineering (TE) to improve network performance by routing traffic in desired ways. However, existing approaches to TE, often, are unable to achieve both high performance and reliability simultaneously.

This paper proposes SMORE, a semi-oblivious TE system based on the insight that the set of forwarding paths has an out-sized impact on the quality of a TE system. SMORE combines oblivious routing for static path selection with dynamic rate adaptation. The paths used by SMORE are low-stretch (ensuring low latency), diverse (ensuring robustness) and enable load-balancing naturally. Dynamic rate adaptation ensures good performance as demands change without incurring overheads related to churn. Through extensive evaluation, we show that SMORE achieves near-optimal performance while ensuring good reliability in practical settings. Based on work published at [1].

[1] Praveen Kumar, Yang Yuan, Chris Yu, Nate Foster, Robert Kleinberg, Petr Lapukhov, Chiun Lin Lim, and Robert Soulé. 2018. Semi-Oblivious Traffic Engineering: The Road Not Taken. In Proceedings of the 15th USENIX Symposium on Networked Systems Design and Implementation (NSDI '18). USENIX Association. 157-170.
\end{abstract}

\footnotetext{
Permission to make digital or hard copies of part or all of this work for personal or classroom use is granted without fee provided that copies are not made or distributed for profit or commercial advantage and that copies bear this notice and the full citation on the first page. Copyrights for third-party components of this work must be honored. For all other uses, contact the owner/author(s).

ANRW'18, July 16, 2018, Montreal, QC, Canada

(C) 2018 Copyright held by the owner/author(s).

ACM ISBN 978-1-4503-5585-8/18/07.

https://doi.org/10.1145/3232755.3232781
}

\author{
Robert Soulé \\ Università della Svizzera italiana \\ robert.soule@usi.ch
}

\section{ACM Reference Format:}

Praveen Kumar, Yang Yuan, Chris Yu, Nate Foster, Robert Kleinberg, Petr Lapukhov, Chiun Lin Lim, and Robert Soulé. 2018. SemiOblivious Traffic Engineering with SMORE. In ANRW '18: Applied Networking Research Workshop, July 16, 2018, Montreal, QC, Canada. ACM, New York, NY, USA, 1 page. https://doi.org/10. $1145 / 3232755.3232781$ 